\title{
Pseudoprogression of vestibular schwannomas after fractionated stereotactic radiation therapy
}

\author{
Fazilat F. Mohammed • Michael L. Schwartz • \\ Alexander Lightstone • David J. Beachey • May N. Tsao
}

Received: 7 November 2012 / Accepted: 20 November 2012 /Published online: 30 November 2012

(C) Springer-Verlag Berlin Heidelberg 2012

\begin{abstract}
Objectives Local tumor control after radiation of vestibular schwannomas (VS) is assessed by follow-up magnetic resonance imaging (MRI) of the brain. Transient increases in tumor volume on MRI due to radiation can mimic tumor progression but are related to radiation treatment effects known as "pseudoprogression". Our study aims to determine the presence and impact of pseudoprogression in VS patients treated with fractionated stereotactic radiotherapy. Methods We analyzed 18 patients with VS treated with fractionated radiotherapy with at least 24 months of follow-up with T1-weighted gadolinium-enhanced MRIs. Mean follow-up time was 42 months. Pre- and posttreatment tumor volumes were calculated using OsiriX ${ }^{\circledR}$ software. Events were defined as volume changes $\geq 10 \%$. Results Tumor control rate was $94 \%$. Forty-four percent of tumors demonstrated increases in volume on follow-up, but all but one patient had transient increases in volume with subsequent regression. Mean tumor volume increased by $35 \%$ with the average time to regression of 24 months. Adverse side effects were more frequent in patients demonstrating pseudoprogression. There were no differences in outcomes based on pre-treatment tumor size or cystic-type versus solid-type tumors.

Conclusions Fractionated stereotactic radiation therapy is an effective treatment for VS. Follow-up imaging with T1-
\end{abstract}

F. F. Mohammed · A. Lightstone · D. J. Beachey • M. N. Tsao ( $\square)$ Department of Radiation Oncology,

Odette Cancer Centre, University of Toronto,

2075 Bayview Ave., Toronto,

Ontario M5N 3M5, Canada

e-mail: may.tsao@sunnybrook.ca

M. L. Schwartz

Division of Neurosurgery, Sunnybrook Health

Sciences Centre, University of Toronto,

Toronto, Ontario, Canada weighted gadolinium-enhanced MRI demonstrated pseudoprogression in a third of patients occurring up to 36 months post-treatment before regression. Therefore, repeat imaging and clinical follow-up of VS should be done to rule out pseudoprogression and avoid unnecessary salvage treatment.

Keywords Vestibular schwannoma · Acoustic neuroma · Pseudoprogression - Fractionated stereotactic radiotherapy . Adverse effects

\section{Introduction}

Vestibular schwannomas (VS), also known as acoustic neuromas, are benign tumors of the vestibulocochlear nerve that account for approximately $8 \%$ of intracranial tumors [1]. These tumors are slow growing and are often diagnosed once they become large enough to cause hearing loss, tinnitus, or vertigo. Treatment options for enlarging VS include surgery, stereotactic radiosurgery, and fractionated stereotactic radiotherapy [1]. Microsurgery is effective but is associated with facial nerve dysfunction in up to $27 \%$ of patients [2]. In order to avoid this morbidity, single-session radiosurgery has been used with good results with reported local control rates of 93-100\% [3-7]. The limitations of radiosurgery include increased toxicity associated with the treatment of larger tumors. Most recently, Hayhurst et al. demonstrated that treatment volume was the most important predictor of adverse radiation effects with a threshold of $5 \mathrm{~cm}^{3}$, above which adverse effects were much more frequent [8]. Alternatively, fractionated radiotherapy has been successfully used for larger tumors and where toxicity of high-dose single-fraction radiation or preservation of serviceable hearing is of concern [9-12].

Serial magnetic resonance imaging (MRI) after radiation treatment usually shows a gradual decrease in tumor volume over time and is currently used to assess tumor control. 
Changes in tumor size and volume over time are used to assess responses and failures; thus, understanding these radiographic changes is important for clinical decision making. The phenomenon of "pseudoprogression" or transient increases in tumor volume that mimic tumor progression but are due to treatment effect has been observed in patients with VS treated with radiation [8, 13-16]. We conducted an institutional study to report the presence and impact of pseudoprogression in VS treated with fractionated stereotactic radiation.

\section{Methods}

Patients

We identified 36 patients at our center with VS treated with fractionated stereotactic radiation therapy from 2002-2009. Patients with at least 24 months of follow-up using serial T1weighted gadolinium-enhanced MRIs to assess tumor response were included in the study. A total of 18 patients treated were eligible and analyzed. Eighteen patients were excluded because of inadequate follow-up or lack of available imaging. Patients with bilateral VS were included but only received unilateral treatment for growing or symptomatic VS. Approval for this study was granted by the Research Ethics Board of the Sunnybrook Health Sciences Centre.

Patient characteristics are summarized in Table 1. Mean age at treatment was 66 years (range, $48-82$ years). Five males and 13 females were treated. One patient had bilateral VS, but only one was treated. Nine patients had solid-type VS, and 9 had cystic-type. Pre-treatment tumor volumes ranged from 0.48 to $12.60 \mathrm{~cm}^{3}$ (mean $4.42 \mathrm{~cm}^{3}$ ). Eight patients had pretreatment tumor volume greater than $5 \mathrm{~cm}^{3}(44 \%)$, while 10 patients $(56 \%)$ had less than $5 \mathrm{~cm}^{3}$. Patients were treated with fractionated stereotactic radiation therapy due to large tumor size. Of the patients with small tumors, two were treated with fractionated radiotherapy prior to our institution obtaining GammaKnife, and the remainder was due to patient choice or in order to decrease the chance of hearing loss in patients with baseline bilateral hearing impairment. No patient had previous surgery or radiation.

Treatment and follow-up

Stereotactic fractionated radiation therapy was delivered using 6-MV beams using a linear accelerator and the GillThomas-Cosman relocatable stereotactic head frame for immobilization. Gross tumor volumes (GTVs) were delineated with both $\mathrm{CT}$ and thin-slice $(1.5 \mathrm{~mm}) \mathrm{T} 1$-weighted gadolinium-enhanced MRI simulation images. A 2-mm margin was added to the GTV for planning target volume (PTV). Radiation of 5,000 cGy in 25 fractions was prescribed to the PTV. Routine follow-up occurred at 6 months,
Table 1 Patient characteristics

\begin{tabular}{ll}
\hline Characteristics & \\
\hline Total number of patients & 18 \\
Mean age (range) & 66 years $(48-82$ years $)$ \\
Number of males & $5(28 \%)$ \\
Number of females & $13(72 \%)$ \\
Mean pre-treatment volume (range) & $4.42 \mathrm{~cm}^{3}\left(0.48-12.6 \mathrm{~cm}^{3}\right)$ \\
$\begin{array}{c}\text { Number of patients with pre-treatment } \\
\quad \text { volume }<5 \mathrm{~cm}^{3}\end{array}$ & $10(56 \%)$ \\
$\begin{array}{c}\text { Number of patients with pre-treatment } \\
\quad \text { volume }>5 \mathrm{~cm}^{3}\end{array}$ & $8(44 \%)$ \\
Number of patients with solid tumors & $9(50 \%)$ \\
Number of patients with cystic tumors & $9(50 \%)$
\end{tabular}

12 months, and then once yearly thereafter. Follow-up imaging was done with $\mathrm{T} 1$ - and T2-weighted pre- and postgadolinium-enhanced MRIs with each follow-up visit. Mean follow-up time was 42 months.

Volume analysis

Pre- and post-treatment tumor volumes were calculated using OsiriX®, a DICOM image processing software program. T1weighted gadolinium-enhanced images were imported into Osiri $\mathrm{X}{ }^{\circledR}$, and tumors were manually contoured slice by slice for each MRI study. Three-dimensional renderings were generated, and volumes were calculated from the outlined target. Tumor volumes were calculated for each follow-up MRI scan. Events were defined as an increase or decrease in volume $\geq 10 \%$.

\section{Adverse side effects}

Adverse effects were recorded by reviewing patient clinical charts. Adverse side effects were defined as a new facial nerve palsy, trigeminal nerve dysfunction, new-onset ataxia, or symptoms of increased edema requiring treatment with steroids.

\section{Statistical analysis}

Paired $t$ test was used to compare differences in pseudoprogression in patients with tumor volumes greater than $5 \mathrm{~cm}^{3}$ versus those with tumors less than $5 \mathrm{~cm}^{3}$, cystictype versus solid-type VS, and the frequency of adverse side effects in pseudoprogressers versus regressors.

\section{Results}

Tumor volume changes

All patient tumor volume changes are illustrated in Fig. 1a. Figure $1 \mathrm{~b}$ summarizes the outcomes in our series. Eight out 
Fig. 1 a Tumor volume changes measured from sequential MRIs after fractionated stereotactic radiation therapy. Each line represents an individual patient's tumor volume over time after treatment compared to pre-treatment volumes (0 months). b Summary of tumor response to fractionated stereotactic radiation therapy

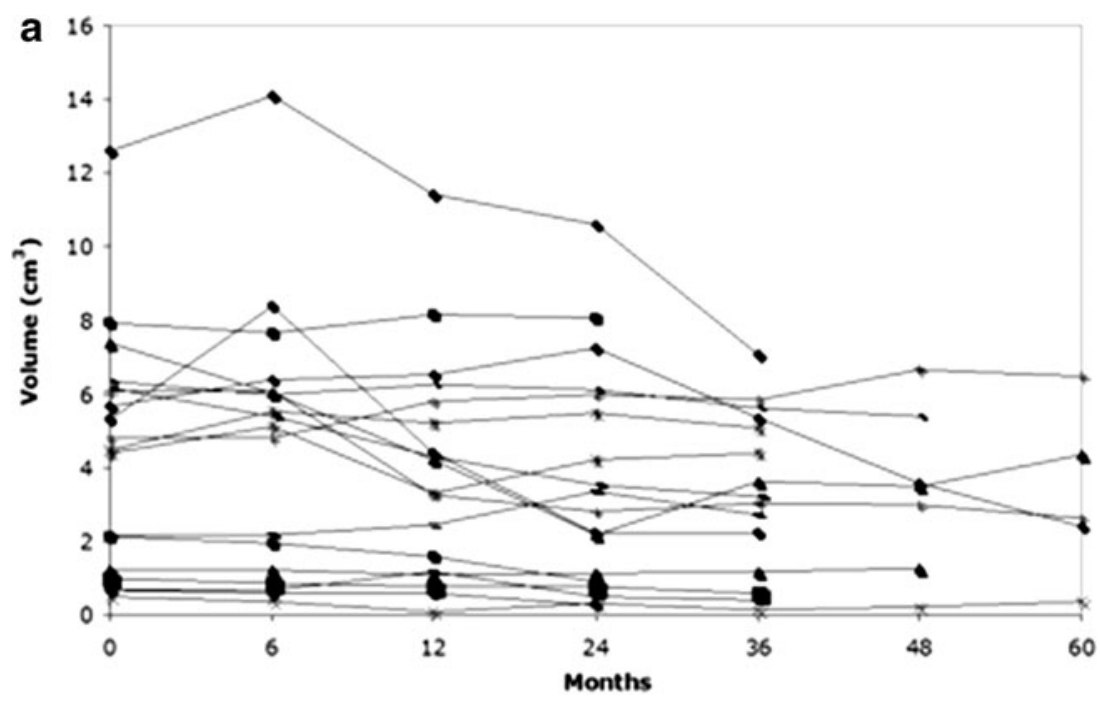

b

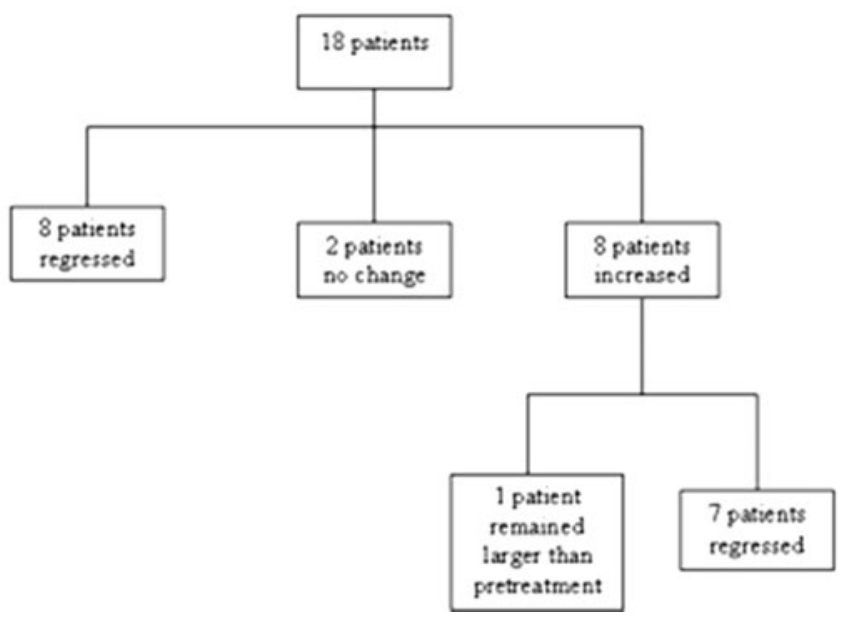

of $18(44 \%)$ patient tumors showed continuous regression in tumor volume following treatment during follow-up imaging. Two of $18(11 \%)$ patient tumors did not demonstrate any significant change in volume. Eight of 18 patients (44\%) demonstrated a significant increase in tumor volume during at least one time point during follow-up imaging. Seven of 18 (39\% of all patients) showed transient increases in tumor volume with eventual tumor regression, (i.e., pseudoprogession) while one (5.6\% of all patients) did not show resolution of the increase in volume at the time of analysis. This patient continues to be followed with observation (currently at 36 months post-treatment) due to slow or minimal growth and with the absence of neurological symptoms. Tumor control rate was $94 \%$ (17/18 patients).

Figure 2 illustrates axial slices from an MRI of a patient demonstrating pseudoprogression after treatment. The mean increase in tumor volume post-treatment was $35 \%$ (range $12-58 \%$ ). In patients with pseudoprogression, peak tumor swelling occurred on average at 18 months post-treatment (range of 6-24 months). The average time for these tumors to regress was 24 months (range of 12-36 months).
Effect of pre-treatment tumor volume

Table 2 compares patients based on pre-treatment volumes. Ten of 18 patients had pre-treatment volumes less than $5 \mathrm{~cm}^{3}$, while eight had pre-treatment volumes greater than $5 \mathrm{~cm}^{3}$. The patient who had continued progression of tumor volume had a pre-treatment volume of less than $5 \mathrm{~cm}^{3}$. The frequency of pseudoprogression or frequency of adverse effects was not statistically different between larger versus smaller tumors. There was no difference in the percentage of volume increase in pseudoprogressors that were greater than $5 \mathrm{~cm}^{3}$ versus those with pre-treatment volumes less than $5 \mathrm{~cm}^{3}$ ( $p=0.83$; Fig. 3a).

\section{Cystic-type versus solid-type VS}

Table 3 compares patients with cystic-type VS to those with solid tumors. Eight patients had cystic-type tumors, while ten had solid-type tumors. Four of the eight patients $(50 \%)$ with cystic-type tumor demonstrated pseudoprogression, while only three of the ten (30\%) solid-type VS showed 
Fig. 2 Example of pseudoprogression with transient tumor volume increases demonstrated on T1weighted gadolinium-enhanced magnetic resonance images of a patient with right-sided vestibular schwannoma preirradiation $\left(\mathbf{a}, 5.7 \mathrm{~cm}^{3}\right)$, 24 months post-irradiation (b, $\left.7.3 \mathrm{~cm}^{3}\right)$, and 60 months postirradiation $\left(\mathbf{c}, 2.4 \mathrm{~cm}^{3}\right)$
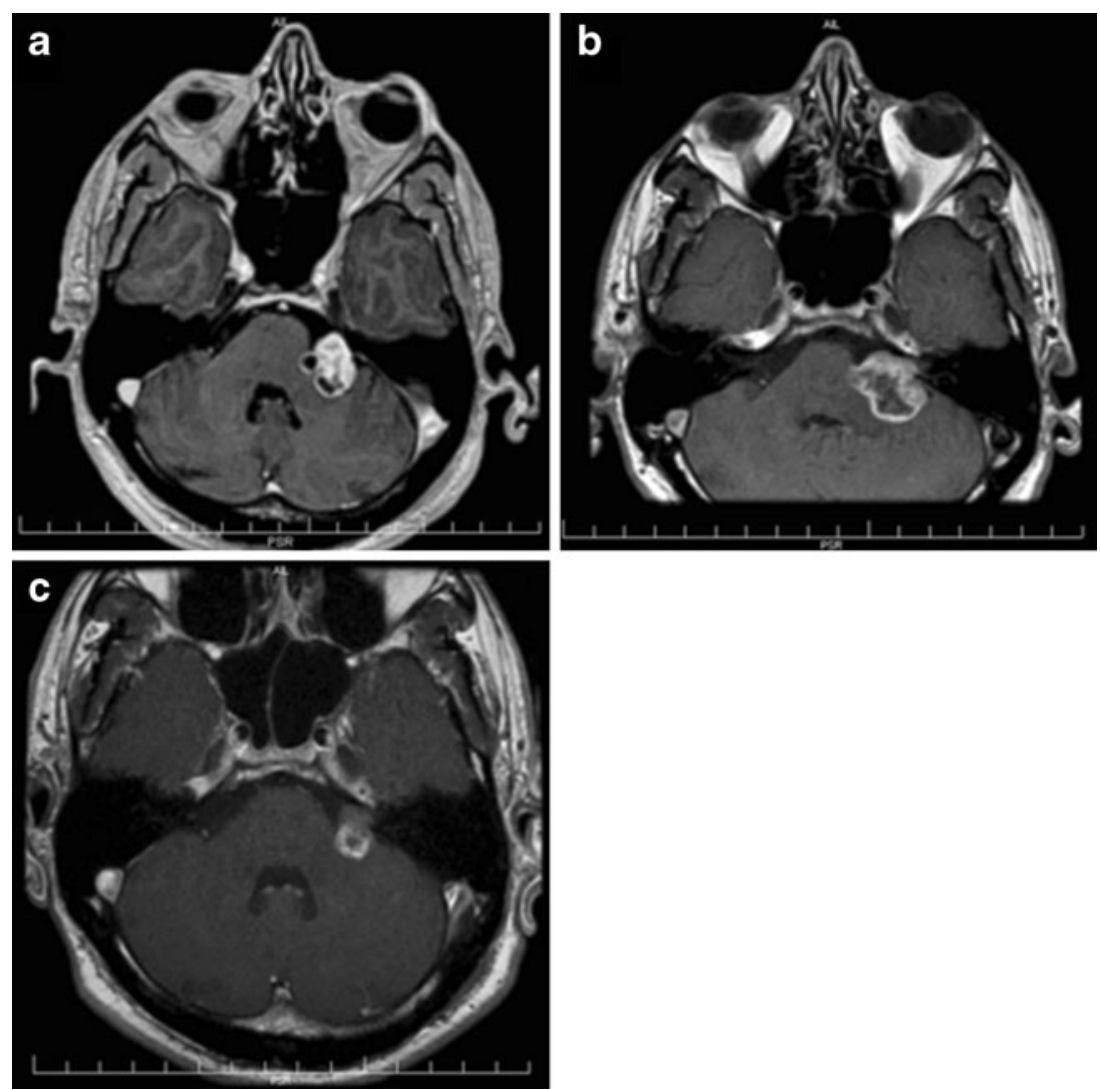

pseudoprogression, although this difference did not reach statistical significance $(p=0.22)$. There was no difference in the mean percentage of volume increase or frequency of adverse effects between the two types of tumors.

Adverse side effects observed in our series

A review of clinical charts revealed that 6 of 18 patients (33\%) reported an adverse side effect upon follow-up. In these six patients, the most frequent neurological symptom was new-onset ataxia (three patients). Two patients reported trigeminal dysfunction (paresthesia in the V2 distribution), and one patient had increasing headaches and nausea/vomiting with increased cerebral edema on imaging. Only one patient (reporting headaches with increased edema on imaging) was offered treatment with steroids, but the patient declined steroids. Four of the six patients with adverse effects were in the group demonstrating pseudoprogression
(57\% of all pseudoprogressors), while only two patients were in the group that had no pseudoprogression $(25 \%$ of all regressors; Fig. 3b). This was not statistically significant $(p=0.23)$. The one patient who did not show resolution of tumor volume progression did not report any adverse effects. All adverse side effects were temporary with no permanent dysfunction.

\section{Discussion}

In this retrospective study, we investigated the outcome of fractionated stereotactic radiation therapy in the treatment of VS. Figure 1b summarizes the outcomes observed in our series. Overall, tumor response was comparable to published results [1, 9-16] with $94 \%$ of tumors being controlled. However, there was a significant proportion of patients $(39 \%)$ who demonstrated transient increases in
Table 2 Comparison of outcomes between patients with tumors greater than $5 \mathrm{~cm}^{3}$ versus those with tumors less than $5 \mathrm{~cm}^{3}$

\begin{tabular}{lll}
\hline & $\begin{array}{l}\text { Tumors }>5 \mathrm{~cm}^{3} \\
n=8\end{array}$ & $\begin{array}{l}\text { Tumors }<5 \mathrm{~cm}^{3} \\
n=10\end{array}$ \\
\hline Frequency of pseudoprogression & $3(38 \%)$ & $4(40 \%)$ \\
Mean percentage of transient tumor volume & $15 \%$ & $12 \%$ \\
Frequency of adverse side effects & $3(38 \%)$ & $4(40 \%)$ \\
\hline
\end{tabular}




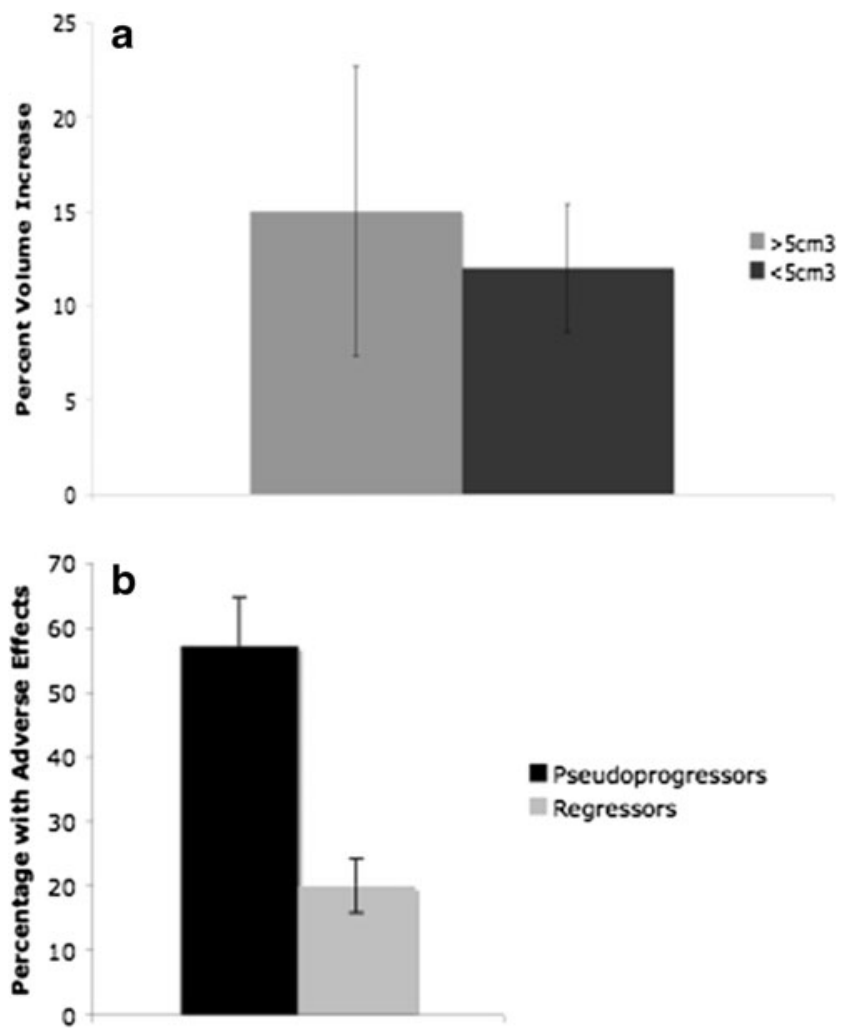

Fig. 3 a Mean \pm standard deviation of the percentage increase in tumor volume in pre-treatment tumor volumes of less than $5 \mathrm{~cm}^{3} . p$ value $=0.827$. b Frequency of adverse side effects in patients whose tumors demonstrated pseudoprogression (pseudoprogressors) compared to patients whose tumors regressed without a transient increase in tumor volume (regressors). $p$ value $=0.23$

tumor volume on MRI, or "pseudoprogression" before eventual tumor regression. This transient change is likely due to radiation effect on the tumor and does not represent actual tumor growth. This phenomenon is well recognized to occur in patients with gliomas treated with fractionated radiation therapy [17]. More recently, several reports have emerged demonstrating transient tumor volume increases in VS treated with stereotactic radiosurgery $[8,13-16]$.

To our knowledge, there is only one other report in the literature by Shirato et al. of transient tumor volume increases after fractionated radiation therapy for VS [13]. Our series adds to the literature demonstrating a significant proportion of patients with VS treated with fractionated stereotactic radiation therapy who undergo pseudoprogression. Shirato et al. also compared pseudoprogression between cystic-type and solid-type VS showing that cystic tumors have an increased frequency of transient volume increases. Our study shows a trend towards increased frequency of pseudoprogression in cystic-type VS, but this did not reach statistical significance likely due to our small sample size.

Overall, we found that patients can demonstrate pseudoprogression up to 24 months, with eventual regression taking up to 36 months. This is also in agreement with the results by Shirato et al. [13]. Thus, we believe that any increase in tumor volume within the first 2 years of treatment, particularly with cystic-type VS, should be observed with serial imaging to rule out pseudoprogression before undergoing salvage treatment, unless there is significant symptomatic mass effect (not responding to steroids) and requiring urgent surgical decompression.

The incidence of adverse side effects in our patient population appears comparable to what is reported in the literature for VS treated with radiation [18, 19]. Trigeminal dysfunction after single-session radiosurgery has been reported to occur at rates of $0-33 \%$ depending on tumor size and peripheral dose delivered [10]. The rate of trigeminal dysfunction in our fractionated radiotherapy series was $11 \%$. Our series showed no permanent adverse effects with the overall transient worsening of neurologic symptoms in $33 \%(6 / 18)$ of patients. We also observed that adverse effects were more common in patients with pseudoprogression likely from a transient increase in pressure on adjacent normal tissue. This was not statistically significant likely due to our small sample size.

The rate of adverse effects can vary widely depending on tumor size and dose delivered. The study by Shirato et al. [13] using fractionated radiation therapy reported a $31 \%$ complication rate. This is comparable to our study; however, they only included tumors $<3.2 \mathrm{~cm}^{3}$. Hayhurst et al. identified a size threshold of $5 \mathrm{~cm}^{3}$, above which adverse effects became much more frequent after single-session radiosurgery with a $75 \%$ complication rate compared to less than $37.5 \%$ in smaller tumors [8]. We examined the effect of tumor size on the outcome of VS after fractionated stereotactic radiotherapy and found no difference in the rates of pseudoprogression or frequency of adverse effects in patients with tumors greater than $5 \mathrm{~cm}^{3}$ (Table 1). These results suggest that patients with VS greater than $5 \mathrm{~cm}^{3}$ may be considered for fractionated stereotactic radiation therapy
Table 3 Comparison of outcomes between patients with cystic tumors versus solid tumors

\begin{tabular}{lll}
\hline & $\begin{array}{l}\text { Cystic tumors } \\
n=8\end{array}$ & $\begin{array}{l}\text { Solid tumors } \\
n=10\end{array}$ \\
\hline $\begin{array}{l}\text { Frequency of pseudoprogression } \\
\text { Mean percentage of transient tumor volume }\end{array}$ & $4(50 \%)$ & $3(30 \%)$ \\
Frequency of adverse side effects & $31 \%$ & $39 \%$ \\
\hline
\end{tabular}


rather than single-session radiosurgery. This would achieve comparable local control rates but with a decreased incidence of adverse effects in this patient population. Patients with tumors greater than $13 \mathrm{~cm}^{3}$ and/or with significant mass effect are generally referred for surgical resection at our center rather than radiation.

The limitations of our study include the small number of patients in our analysis and short follow-up period of these patients. Also, we did not do a direct or randomized comparison to GammaKnife-treated patients. Larger randomized studies comparing rates of pseudoprogression and adverse side effects in patients treated with single-session radiosurgery versus fractionated radiotherapy may demonstrate which patients would benefit from single versus fractionated treatment.

\section{Conclusions}

Our study demonstrates that serial MRIs of patients with VS treated with fractionated stereotactic radiation therapy can show transient increases in tumor volume due to treatment effect, or pseudoprogression. Adverse effects are more common in patients with pseudoprogression but are not more frequent in larger tumors using this treatment technique. In patients with asymptomatic VS enlargement after radiotherapy, follow-up serial MRI should be performed to differentiate pseudoprogression from true tumor progression.

Conflict of interest On behalf of all authors, the corresponding author states that there is no conflict of interest.

\section{References}

1. Murphy ES, Suh JH (2011) Radiotherapy for vestibular schwannomas: a critical review. Int J Radiat Oncol Biol Phys 79:985-997

2. Samii M, Matthies C (1997) Management of 1000 vestibular schwannomas (acoustic neuromas): the facial nerve preservation and restitution of function. Neurosurgery 40:684-694

3. Lunsford LD, Niranjan A, Flickinger JC et al (2005) Radiosurgery of vestibular schwannomas: summary of experience in 829 cases. $\mathrm{J}$ Neurosurg 102(Suppl):195-199
4. Miller RC, Foote RL, Coffey RJ et al (1999) Decrease in cranial nerve complications after radiosurgery for acoustic neuromas: a prospective study of dose and volume. Int J Radiat Oncol Biol Phys 43:305-311

5. Flickinger JC, Kondziolka D, Niranjan A et al (2001) Results of acoustic neuroma radiosurgery: an analysis of 5 years' experience using current methods. J Neurosurg 94:1-6

6. Spiegelmann R, Lidar Z, Gofman J et al (2001) Linear accelerator radiosurgery for vestibular schwannoma. J Neurosurg 94:7-13

7. Foote KD, Friedman WA, Buatti JM et al (2001) Analysis of risk factors associated with radiosurgery for vestibular schwannoma. $\mathrm{J}$ Neurosurg 95:440-449

8. Hayhurst C, Monsalves E, Bernstein M et al (2012) Predicting nonauditory adverse radiation effects following radiosurgery for vestibular schwannoma: a volume and dosimetric analysis. Int J Radiat Oncol Biol Phys 82(5):2041-2046

9. Szumacher E, Schwartz ML, Tsao M et al (2002) Fractionated stereotactic radiotherapy for the treatment of vestibular schwannomas: combined experience of the Toronto-Sunnybrook Regional Cancer Centre and the Princess Margaret Hospital. Int J Radiat Oncol Biol Phys 53:987-91

10. Kalapurakal JA, Silverman CL, Akhtar N et al (1999) Improved trigeminal and facial nerve tolerance following fractionated stereotactic radiotherapy for large acoustic neuromas. Br J Radiol 72:1202-1207

11. Selch MT, Pedroso A, Lee SP et al (2004) Stereotactic radiotherapy for the treatment of acoustic neuromas. J Neurosurg 101(suppl 3):362-372

12. Combs SE, Volk S, Schulz-Ertner D et al (2005) Management of acoustic neuromas with fractionated stereotactic radiotherapy (FSRT): long-term results in 106 patients treated in a single institution. Int J Radiat Oncol Biol Phys 63:75-81

13. Shirato H, Sakamoto T, Takeichi N et al (2000) Fractionated stereotactic radiotherapy for vestibular schwannoma (VS): comparison between cystic-type and solid-type VS. Int J Radiat Oncol Biol Phys 48:1395-1401

14. Nagano O, Higuchi Y, Serizawa T et al (2008) Transient expansion of vestibular schwannoma following stereotactic radiosurgery. $\mathrm{J}$ Neurosurg 109:811-6

15. Meijer OW, Weijmans EJ, Knol DL et al (2008) Tumor-volume changes after radiosurgery for vestibular schwannoma: implications for follow-up MR imaging protocol. AJNR Am J Neuroradiol 29:906-10

16. Nakamura H, Jokura H, Takahashi K et al (2000) Serial follow-up MR imaging after gamma knife radiosurgery for vestibular schwannoma. AJNR Am J Neuroradiol 21:1540-6

17. Brandsma D, Stalpers L, Taal W et al (2008) Clinical features, mechanisms, and management of pseudoprogression in malignant gliomas. Lancent Oncol 9:453-61

18. Sughrue ME, Yang I, Han SJ et al (2009) Non-audiofacial morbidity after gamma knife surgery for vestibular schwannoma. Neurosurg Focus 27:E4

19. Flickinger JC, Lunsford LD, Linskey ME et al (1993) Gamma knife radiosurgery for acoustic tumors: multivariate analysis of four year results. Radiother Oncol 27:91-98 